\title{
Electromagnetic Compatibility Engineering
}

Henry W. Ott

Henry Ott Consultants

(10) WILEY

A JOHN WILEY \& SONS, INC., PUBLICATION 


\section{CONTENTS}

$\begin{array}{ll}\text { Preface } & \text { xxiii }\end{array}$

PART 1 EMC THEORY 1

1. Electromagnetic Compatibility 3

1.1 Introduction 3

1.2 Noise and Interference 3

1.3 Designing for Electromagnetic Compatibility 4

1.4 Engineering Documentation and EMC 6

1.5 United States' EMC Regulations 6

1.5.1 FCC Regulations $\quad 6$

1.5.2 FCC Part 15, Subpart B 8

1.5.3 Emissions 11

1.5.4 Administrative Procedures 14

$\begin{array}{lll}1.5 .5 & \text { Susceptibility } & 17\end{array}$

$\begin{array}{ll}\text { 1.5.6 Medical Equipment } & 17\end{array}$

$\begin{array}{lll}1.5 .7 & \text { Telecom } & 18\end{array}$

$\begin{array}{lll}1.5 .8 \text { Automotive } & 19\end{array}$

1.6 Canadian EMC Requirements 19

1.7 European Union's EMC Requirements 20

1.7.1 Emission Requirements 20

1.7.2 Harmonics and Flicker $\quad 22$

1.7.3 Immunity Requirements 23

1.7.4 Directives and Standards 23

1.8 International Harmonization 26

$\begin{array}{lll}1.9 & \text { Military Standards } & 27\end{array}$ 
1.10 Avionics 28

1.11 The Regulatory Process 30

1.12 Typical Noise Path 30

1.13 Methods of Noise Coupling 31

1.13.1 Conductively Coupled Noise 31

1.13.2 Common Impedance Coupling 32

1.13.3 Electric and Magnetic Field Coupling 33

1.14 Miscellaneous Noise Sources 33

1.14.1 Galvanic Action 33

1.14.2 Electrolytic Action $\quad 35$

1.14.3 Triboelectric Effect 35

1.14.4 Conductor Motion 36

1.15 Use of Network Theory 36

Summary 38

Problems 39

References 41

Further Reading $\quad 42$

2. Cabling 44

2.1 Capacitive Coupling 45

2.2 Effect of Shield on Capacitive Coupling 48

2.3 Inductive Coupling 52

2.4 Mutual Inductance Calculations 54

2.5 Effect of Shield on Magnetic Coupling 56

2.5.1 Magnetic Coupling Between Shield and Inner Conductor 58

2.5.2 Magnetic Coupling-Open Wire to Shielded Conductor 61

2.6 Shielding to Prevent Magnetic Radiation 64

2.7 Shielding a Receptor Against Magnetic Fields 67

2.8 Common Impedance Shield Coupling 69

$\begin{array}{lll}2.9 & \text { Experimental Data } & 70\end{array}$

2.10 Example of Selective Shielding $\quad 74$

2.11 Shield Transfer Impedance 75

2.12 Coaxial Cable Versus Twisted Pair 75 
2.13 Braided Shields $\quad 79$

2.14 Spiral Shields $\quad 81$

2.15 Shield Terminations $\quad 84$

$\begin{array}{lll}2.15 .1 \text { Pigtails } & 84\end{array}$

2.15.2 Grounding of Cable Shields 88

2.16 Ribbon Cables 94

2.17 Electrically Long Cables 96

Summary $\quad 96$

Problems $\quad 98$

References 103

Further Reading 104

3. Grounding 106

3.1 AC Power Distribution and Safety Grounds 107

3.1.1 Service Entrance 108

3.1.2 Branch Circuits 109

3.1.3 Noise Control 111

3.1.4 Earth Grounds $\quad 114$

3.1.5 Isolated Grounds $\quad 116$

3.1.6 Separately Derived Systems 118

$\begin{array}{ll}\text { 3.1.7 Grounding Myths } & 119\end{array}$

3.2 Signal Grounds 120

3.2.1 Single-Point Ground Systems 124

3.2.2 Multipoint Ground Systems 126

3.2.3 Common Impedance Coupling 128

3.2.4 Hybrid Grounds 130

3.2.5 Chassis Grounds 131

3.3 Equipment/System Grounding 132

3.3.1 Isolated Systems 133

3.3.2 Clustered Systems 133

3.3.3 Distributed Systems 140

3.4 Ground Loops 142

3.5 Low-Frequency Analysis of Common-Mode Choke 147

3.6 High-Frequency Analysis of Common-Mode Choke 152

3.7 Single Ground Reference for a Circuit 154 
X CONTENTS

$\begin{array}{ll}\text { Summary } & 155\end{array}$

Problems 156

References 157

$\begin{array}{ll}\text { Further Reading } & 157\end{array}$

4. Balancing and Filtering 158

$\begin{array}{lll}4.1 & \text { Balancing } & 158\end{array}$

4.1.1 Common-Mode Rejection Ratio 161

4.1.2 Cable Balance 165

4.1.3 System Balance 166

4.1.4 Balanced Loads 166

$\begin{array}{lll}4.2 & \text { Filtering } & 174\end{array}$

4.2.1 Common-Mode Filters 174

4.2.2 Parasitic Effects in Filters 177

4.3 Power Supply Decoupling 178

4.3.1 Low-Frequency Analog Circuit Decoupling 183

4.3.2 Amplifier Decoupling 185

4.4 Driving Capacitive Loads 186

4.5 System Bandwidth 188

4.6 Modulation and Coding 190

$\begin{array}{ll}\text { Summary } & 190\end{array}$

Problems 191

References 192

Further Reading 193

5. Passive Components 194

$\begin{array}{lll}5.1 \text { Capacitors } & 194\end{array}$

5.1.1 Electrolytic Capacitors $\quad 195$

5.1.2 Film Capacitors 197

5.1.3 Mica and Ceramic Capacitors 198

5.1.4 Feed-Through Capacitors 200

5.1.5 Paralleling Capacitors 202

5.2 Inductors 203

5.3 Transformers 204

$\begin{array}{lll}5.4 & \text { Resistors } & 206\end{array}$

5.4.1 Noise in Resistors 207 
CONTENTS $\mathbf{x i}$

5.5 Conductors 208

5.5.1 Inductance of Round Conductors 209

5.5.2 Inductance of Rectangular Conductors 210

5.5.3 Resistance of Round Conductors 211

5.5.4 Resistance of Rectangular Conductors 213

5.6 Transmission Lines 215

5.6.1 Characteristic Impedance 217

5.6.2 Propagation Constant 220

5.6.3 High-Frequency Loss 221

5.6.4 Relationship Among $C, L$ and $\varepsilon_{r}$. 224

5.6.5 Final Thoughts 225

5.7 Ferrites 225

Summary 233

Problems 234

References $\quad 237$

Further Reading 237

6. Shielding 238

6.1 Near Fields and Far Fields 238

6.2 Characteristic and Wave Impedances 241

6.3 Shielding Effectiveness 243

6.4 Absorption Loss 245

6.5 Reflection Loss 249

6.5.1 Reflection Loss to Plane Waves 252

6.5.2 Reflection Loss in the Near Field 253

6.5.3 Electric Field Reflection Loss 254

6.5.4 Magnetic Field Reflection Loss 255

6.5.5 General Equations for Reflection Loss 256

6.5.6 Multiple Reflections in Thin Shields 256

6.6 Composite Absorption and Reflection Loss 257

$\begin{array}{lll}\text { 6.6.1 Plane Waves } & 257\end{array}$

6.6.2 Electric Fields $\quad 258$

6.6.3 Magnetic Fields 259

$\begin{array}{lll}6.7 & \text { Summary of Shielding Equations } & 260\end{array}$

6.8 Shielding with Magnetic Materials 260

6.9 Experimental Data 265 
xii CONTENTS

$\begin{array}{lll}6.10 & \text { Apertures } & 267\end{array}$

6.10.1 Multiple Apertures $\quad 270$

6.10.2 Seams 273

6.10.3 Transfer Impedance $\quad 277$

6.11 Waveguide Below Cutoff 280

6.12 Conductive Gaskets 282

6.12.1 Joints of Dissimilar Metals 283

6.12.2 Mounting of Conductive Gaskets 284

6.13 The "IDEAL" Shield 287

6.14 Conductive Windows 288

6.14.1 Transparent Conductive Coatings 288

6.14.2 Wire Mesh Screens 289

6.14.3 Mounting of Windows $\quad 289$

6.15 Conductive Coatings 289

6.15.1 Conductive Paints 291

6.15.2 Flame/Arc Spray 291

6.15.3 Vacuum Metalizing 291

6.15.4 Electroless Plating 292

6.15.5 Metal Foil Linings $\quad 292$

6.15.6 Filled Plastic 293

6.16 Internal Shields 293

6.17 Cavity Resonance 295

6.18 Grounding of Shields 296

Summary 296

Problems $\quad 297$

References 299

$\begin{array}{ll}\text { Further Reading } & 300\end{array}$

7. Contact Protection 302

7.1 Glow Discharges 302

7.2 Metal-Vapor or Arc Discharges 303

7.3 AC Versus DC Circuits 305

7.4 Contact Material 306

7.5 Contact Rating 306

$\begin{array}{ll}7.6 & \text { Loads with High Inrush Currents } \\ 307\end{array}$ 
7.7 Inductive Loads 308

7.8 Contact Protection Fundamentals 310

7.9 Transient Suppression for Inductive Loads 314

7.10 Contact Protection Networks for Inductive Loads 318

$\begin{array}{lll}7.10 .1 C \text { Network } & 318\end{array}$

$\begin{array}{lll}7.10 .2 & R-C \text { Network } & 318\end{array}$

7.10.3 R-C-D Network 321

7.11 Inductive Loads Controlled by a Transistor Switch 322

7.12 Resistive Load Contact Protection 323

7.13 Contact Protection Selection Guide 323

7.14 Examples $\quad 324$

Summary $\quad 325$

Problems $\quad 326$

References $\quad 327$

Further Reading $\quad 327$

8. Intrinsic Noise Sources $\quad 328$

8.1 Thermal Noise 328

8.2 Characteristics of Thermal Noise 332

8.3 Equivalent Noise Bandwidth 334

$\begin{array}{lll}8.4 & \text { Shot Noise } & 337\end{array}$

8.5 Contact Noise 338

8.6 Popcorn Noise 339

8.7 Addition of Noise Voltages 340

8.8 Measuring Random Noise 341

Summary $\quad 342$

Problems 343

References $\quad 345$

Further Reading 345

9. Active Device Noise 346

9.1 - Noise Factor 346

9.2 Measurement of Noise Factor 349 
xiv CONTENTS

9.2.1 Single-Frequency Method

9.2.2 Noise Diode Method

9.3 Calculating $S / N$ Ratio and Input Noise Voltage from Noise Factor

9.4 Noise Voltage and Current Model 353

9.5 Measurment of $V_{n}$ and $I_{n}$

9.6 Calculating Noise Factor and $S / N$ Ratio from $V_{n}-I_{n}$

9.7 Optimum Source Resistance 357

9.8 Noise Factor of Cascaded Stages 360

9.9 Noise Temperature 362

9.10 Bipolar Transistor Noise $\quad 364$

9.10.1 Transistor Noise Factor $\quad 365$

9.10.2 $V_{n}-I_{n}$ for Transistors 367

9.11 Field-Effect Transistor Noise 368

9.11.1 FET Noise Factor $\quad 368$

9.11.2 $V_{n}-I_{n}$ Representation of FET Noise $\quad 370$

9.12 Noise in Operational Amplifiers $\quad 370$

9.12.1 Methods of Specifying Op-Amp Noise 373

9.12.2 Op-Amp Noise Factor 375

Summary $\quad 375$

Problems $\quad 376$

References $\quad 377$

$\begin{array}{ll}\text { Further Reading } & 378\end{array}$

10. Digital Circuit Grounding 379

10.1 Frequency Versus Time Domain 380

10.2 Analog Versus Digital Circuits 380

10.3 Digital Logic Noise 380

10.4 Internal Noise Sources 381

10.5 Digital Circuit Ground Noise 384

10.5.1 Minimizing Inductance 385

10.5.2 Mutual Inductance $\quad 386$

10.5.3 Practical Digital Circuit Ground Systems 388

10.5.4 Loop Area 390 
10.6 Ground Plane Current Distribution and Impedance 391

10.6.1 Reference Plane Current Distribution 392

10.6.2 Ground Plane Impedance $\quad 400$

10.6.3 Ground Plane Voltage 408

10.6.4 End Effects 409

10.7 Digital Logic Current Flow 412

10.7.1 Microstrip Line $\quad 414$

$\begin{array}{lll}\text { 10.7.2 Stripline } & 415\end{array}$

10.7.3 Digital Circuit Current Flow Summary 418

$\begin{array}{ll}\text { Summary } & 419\end{array}$

Problems $\quad 420$

References $\quad 421$

Further Reading $\quad 422$

$\begin{array}{lll}\text { PART } 2 & \text { EMC APPLICATIONS } & 423\end{array}$

11. Digital Circuit Power Distribution 425

11.1 Power Supply Decoupling 425

11.2 Transient Power Supply Currents $\quad 426$

11.2.1 Transient Load Current $\quad 427$

11.2.2 Dynamic Internal Current $\quad 428$

11.2.3 Fourier Spectrum of the Transient Current 429

11.2.4 Total Transient Current 431

11.3 Decoupling Capacitors 431

11.4 Effective Decoupling Strategies 436

11.4.1 Multiple Decoupling Capacitors 437

11.4.2 Multiple Capacitors of the Same Value 437

11.4.3 Multiple Capacitors of Two Different Values 440

11.4.4 Multiple Capacitors of Many Different Values 444

11.4.5 Target Impedance $\quad 445$

11.4.6 Embedded PCB Capacitance 447

11.4.7 Power Supply Isolation 452

11.5 The Effect of Decoupling on Radiated Emissions 454

11.6 Decoupling Capacitor Type and Value 456

11.7 Decoupling Capacitor Placement and Mounting 457

$\begin{array}{lll}11.8 & \text { Bulk Decoupling Capacitors } & 459\end{array}$ 
xvi CONTENTS

11.9 Power Entry Filters 460

Summary 461

Problems 461

References 463

Further Reading 463

12. Digital Circuit Radiation 464

12.1 Differential-Mode Radiation 465

12.1.1 Loop Area 468

12.1.2 Loop Current 468

12.1.3 Fourier Series 468

12.1.4 Radiated Emission Envelope $\quad 470$

12.2 Controlling Differential-Mode Radiation 471

12.2.1 Board Layout $\quad 471$

12.2.2 Canceling Loops 474

12.2.3 Dithered Clocks 475

12.3 Common-Mode Radiation 477

12.4 Controlling Common-Mode Radiation 480

12.4.1 Common-Mode Voltage 481

12.4.2 Cable Filtering and Shielding 482

12.4.3 Separate I/O Grounds 485

12.4.4 Dealing With Common-Mode Radiation Issues 488

Summary $\quad 488$

$\begin{array}{ll}\text { Problems } & 489\end{array}$

References 490

Further Reading 491

13. Conducted Emissions 492

13.1 Power Line Impedance 492

13.1.1 Line Impedance Stabilization Network 494

13.2 Switched-Mode Power Supplies 495

13.2.1 Common-Mode Emissions 498

13.2.2 Differential-Mode Emissions 501

$\begin{array}{ll}\text { 13.2.3 DC-to-DC Converters } & 509\end{array}$

13.2.4 Rectifier Diode Noise $\quad 509$ 
13.3 Power-Line Filters 511

13.3.1 Common-Mode Filtering $\quad 512$

13.3.2 Differential-Mode Filtering 512

13.3.3 Leakage Inductance $\quad 513$

13.3.4 Filter Mounting 516

13.3.5 Power Supplies with Integral Power-Line Filters $\quad 519$

13.3.6 High-Frequency Noise $\quad 520$

13.4 Primary-to-Secondary Common-Mode Coupling 523

13.5 Frequency Dithering 524

13.6 Power Supply Instability 524

13.7 Magnetic Field Emissions $\quad 525$

$13.8 \quad$ Variable Speed Motor Drives $\quad 528$

13.9 Harmonic Suppression 536

13.9.1 Inductive Input Filters $\quad 538$

13.9.2 Active Power Factor Correction 538

13.9.3 AC Line Reactors 539

Summary 541

Problems $\quad 542$

References $\quad 544$

Further Reading 544

14. RF and Transient Immunity 545

14.1 Performance Criteria 545

14.2 RF Immunity 546

14.2.1 The RF Environment 547

14.2.2 Audio Rectification $\quad 548$

14.2.3 RFI Mitigation Techniques 549

$\begin{array}{lll}14.3 & \text { Transient Immunity } & 557\end{array}$

14.3.1 Electrostatic Discharge $\quad 558$

14.3.2 Electrical Fast Transient 558

14.3.3 Lightning Surge $\quad 559$

14.3.4 Transient Suppression Networks $\quad 560$

14.3.5 Signal Line Suppression $\quad 561$

14.3.6 Protection of High-Speed Signal Lines $\quad 564$

14.3.7 Power Line Transient Suppression $\quad 566$

14.3.8 Hybrid Protection Network 570 
xviii CONTENTS

14.4 Power Line Disturbances 572

14.4.1 Power Line Immunity Curve 573

Summary $\quad 575$

Problems 576

References 578

Further Reading $\quad 579$

15. Electrostatic Discharge $\quad 580$

15.1 Static Generation 580

15.1.1 Inductive Charging 583

15.1.2 Energy Storage 585

15.2 Human Body Model 587

15.3 Static Discharge $\quad 589$

15.3.1 Decay Time 590

15.4 ESD Protection in Equipment Design 592

15.5 Preventing ESD Entry 594

15.5.1 Metallic Enclosures 595

15.5.2 Input/Output Cable Treatment 599

15.5.3 Insulated Enclosures $\quad 604$

15.5.4 Keyboards and Control Panels 607

15.6 Hardening Sensitive Circuits 608

$\begin{array}{ll}15.7 & \text { ESD Grounding }\end{array}$

15.8 Nongrounded Products 609

15.9 Field-Induced Upset 610

15.9.1 Inductive Coupling 611

15.9.2 Capacitive Coupling 611

15.10 Transient Hardened Software Design 612

15.10.1 Detecting Errors in Program Flow 613

15.10.2 Detecting Errors in Input/Output 614

15.10.3 Detecting Errors in Memory 616

15.11 Time Windows $\quad 617$

$\begin{array}{ll}\text { Summary } & 617\end{array}$

$\begin{array}{ll}\text { Problems } & 619\end{array}$ 
$\begin{array}{ll}\text { References } & 620\end{array}$

Further Reading $\quad 621$

16. PCB Layout and Stackup $\quad 622$

16.1 General PCB Layout Considerations 622

16.1.1 Partitioning $\quad 622$

16.1.2 Keep Out Zones $\quad 622$

16.1.3 Critical Signals $\quad 623$

16.1.4 System Clocks $\quad 624$

16.2 PCB-to-Chassis Ground Connection 625

16.3 Return Path Discontinuities 626

16.3.1 Slots in Ground/Power Planes $\quad 627$

16.3.2 Split Ground/Power Planes $\quad 628$

16.3.3 Changing Reference Planes 630

16.3.4 Referencing the Top and Bottom of the Same Plane 633

16.3.5 Connectors 634

16.3.6 Ground Fill 634

16.4 PCB Layer Stackup $\quad 635$

16.4.1 One- and Two-Layer Boards 636

16.4.2 Multilayer Boards 637

16.4.3 General PCB Design Procedure 653

Summary $\quad 655$

$\begin{array}{ll}\text { Problems } & 657\end{array}$

References $\quad 658$

$\begin{array}{ll}\text { Further Reading } & 658\end{array}$

17. Mixed-Signal PCB Layout 660

$\begin{array}{lll}17.1 & \text { Split Ground Planes } & 660\end{array}$

17.2 Microstrip Ground Plane Current Distribution 662

17.3 Analog and Digital Ground Pins 665

17.4 When Should Split Ground Planes Be Used? 668

$\begin{array}{lll}17.5 & \text { Mixed Signal ICs } & 669\end{array}$

$\begin{array}{lll}\text { 17.5.1 Multi-Board Systems } & 671\end{array}$

17.6 High-Resolution A/D and D/A Converters 671

$\begin{array}{lll}\text { 17.6.1 Stripline } & 673\end{array}$ 
17.6.2 Asymmetric Stripline

17.6.3 Isolated Analog and Digital Ground Planes

17.7 A/D and D/A Converter Support Circuitry

17.7.1 Sampling Clocks

17.7.2 Mixed-Signal Support Circuitry

17.8 Vertical Isolation

17.9 Mixed-Signal Power Distribution

17.9.1 Power Distribution

17.9.2 Decoupling

17.10 The IPC Problem

Summary

Problems

References

Further Reading

18. Precompliance EMC Measurements

18.1 Test Environment

18.2 Antennas Versus Probes 689

18.3 Common-Mode Currents on Cables $\quad 690$

18.3.1 Test Procedure $\quad 693$

18.3.2 Cautions 693

18.4 Near Field Measurements $\quad 694$

18.4.1 Test Procedure $\quad 695$

18.4.2 Cautions $\quad 696$

18.4.3 Seams and Apertures in Enclosures 697

$\begin{array}{ll}\text { 18.5 Noise Voltage Measurements } & 697\end{array}$

18.5.1 Balanced Differential Probe 698

18.5.2 DC to $1-\mathrm{GHz}$ Probe $\quad 700$

$\begin{array}{ll}\text { 18.5.3 Cautions } & 700\end{array}$

18.6 Conducted Emission Testing 700

18.6.1 Test Procedure 702

18.6.2 Cautions 703

18.6.3 Separating C-M from D-M Noise 704

$\begin{array}{lll}18.7 & \text { Spectrum Analyzers } & 707\end{array}$ 
18.7.1 Detector Functions $\quad 709$

18.7.2 General Test Procedure $\quad 710$

$\begin{array}{lll}18.8 & \text { EMC Crash Cart } & 711\end{array}$

18.8.1 Mitigation Parts List 712

18.9 One-Meter Radiated Emission Measurements $\quad 713$

18.9.1 Test Environment 713

18.9.2 Limits for 1-m Testing 713

18.9.3 Antennas for 1-m Testing 714

$\begin{array}{lll}18.10 & \text { Precompliance Immunity Testing } & 717\end{array}$

$\begin{array}{lll}18.10 .1 & \text { Radiated Immunity } & 717\end{array}$

18.10.2 Conducted Immunity $\quad 720$

18.10.3 Transient Immunity 721

18.11 Precompliance Power Quality Tests 723

$\begin{array}{lll}\text { 18.11.1 Harmonics } & 724\end{array}$

$\begin{array}{lll}18.11 .2 & \text { Flicker } & 725\end{array}$

$\begin{array}{lll}18.12 \text { Margin } & 726\end{array}$

18.12.1 Radiated Emission Margin $\quad 726$

18.12.2 Electrostatic Discharge Margin 727

Summary $\quad 728$

$\begin{array}{ll}\text { Problems } & 729\end{array}$

References $\quad 730$

Further Reading 731

$\begin{array}{ll}\text { APPENDIX } & 733\end{array}$

A. The Decibel 733

A.1 Properties of Logarithms 733

A.2 Using the Decibel for Other than Power Measurements 734

A.3 Power Loss or Negative Power Gain 736

A.4 Absolute Power Level 736

A.5 Summing Powers Expressed in Decibels 738

B. The Ten Best Ways to Maximize the Emission from Your Product $\mathbf{7 4 0}$

C. Multiple Reflections of Magnetic Fields in Thin Shields 743 
xxii CONTENTS

D. Dipoles for Dummies $\quad \mathbf{7 4 6}$

D.1 Basic Dipoles for Dummies 746

D.2 Intermediate Dipoles for Dummies 751

D.3 Advanced Dipoles for Dummies 756

D.3.1 Impedance of a Dipole 756

D.3.2 Dipole Resonance 756

$\begin{array}{lll}\text { D.3.3 Receiving Dipole } & 759\end{array}$

$\begin{array}{lll}\text { D.3.4 Theory of Images } & 759\end{array}$

$\begin{array}{ll}\text { D.3.5 Dipole Arrays } & 761\end{array}$

D.3.6 Very High-Frequency Dipoles 763

Summary $\quad 763$

$\begin{array}{ll}\text { Further Reading } & 764\end{array}$

E. Partial Inductance $\quad \mathbf{7 6 5}$

E.1 Inductance 765

$\begin{array}{lll}\text { E.2 Loop Inductance } & 767\end{array}$

E.2.1 Inductance of a Rectangular Loop 768

$\begin{array}{lll}\text { E.3 Partial Inductance } & 770\end{array}$

E.3.1 Partial Self-Inductance $\quad 771$

E.3.2 Partial Mutual Inductance $\quad 773$

E.3.3 Net Partial-Inductance $\quad 776$

E.3.4 Partial Inductance Applications $\quad 776$

E.3.5 Transmission Line Example 778

E.4 Ground Plane Inductance Measurement Test Setup 780

$\begin{array}{lll}\text { E.5 Inductance Notation } & 785\end{array}$

$\begin{array}{ll}\text { Summary } & 788\end{array}$

$\begin{array}{ll}\text { References } & 788\end{array}$

$\begin{array}{ll}\text { Further Reading } & 789\end{array}$

F. Answers to Problems 790

$\begin{array}{ll}\text { Index } & 825\end{array}$ 